\title{
Your Fuzzy Recovery of TV Guidance Dynamic Image Frame Based on Lifting Wavelet
}

\author{
Huiyong Zhang ${ }^{1}$, Zeqian $\mathrm{Liu}^{1}$, Yiguo $\mathrm{Ji}^{1}$, Manyi Hou ${ }^{1}$, Dong Yang ${ }^{2}$ \\ ${ }^{1}$ Air Force Aviation University, Changchun, 130022, China \\ ${ }^{2}$ Air Force Harbin Flight Academy, Harbin, 150223, China
}

Keywords: TV guidance, lifting wavelet, image blurs, PSF frequency domain estimation.

\begin{abstract}
In view of the TV guided missile terminal guidance phase of visible light image information intra- frame of fuzzy focuses on the characteristics of the low frequency part of image, the lifting wavelet improvement are introduced to the blur image restoration field of terminal guidance phase intra-frame motion. The multi-point averaging PSF estimation method is put forward aiming at the result of the Hough transform. The improved algorithm of fuzzy parameters is proposed considering the thought of using wiener filtering; further improve the effectiveness of the estimation accuracy and estimation. The digital simulation analysis and verification of proposed recovery with adaptive wiener filtering method are done.
\end{abstract}

\section{Introduction}

Because of the TV guided missile seeker active movement, irregular random movement of seeker stabilized platform, and unpredictable of all kinds of battlefield sudden interference, which sometimes cause appearance of intra-frame of fuzzy guidance image information.

For motion blur image frame recovery, the key lies in the acquisition of PSF, the more accurate of obtained PSF, the more effective of motion blurred image restoration. But the conventional frequency domain method based on Fourier transform are greatly influenced by the high frequency noise, and the process of TV guided missile terminal guidance stage exists a lot of visible light white Gaussian noise, and the estimation method of using the current PSF frequency domain will be difficult to ensure the estimation precision, and based on global PSF frequency domain estimation method to deal with large amount of image data, it is hard to meet the need of real time.

\section{TV guidance dynamic fuzzy design method to restore the image intra-frame}

In view of the image motion blur mainly exist in the low frequency part of image of the most energy, draw lessons from the advantages of lifting wavelet and median filtering method, the ascension of wavelet and median filtering method is put forward for handling of dynamic image frame fuzzy TV guided missile, the high frequency sub band characteristics to adaptive wiener filtering method is used to recover, to avoid the influence of high frequency noise and protect the image edge information, improve the quality of image restoration, and improve the real-time processing speed. The method of algorithm process is shown in Fig. 1.

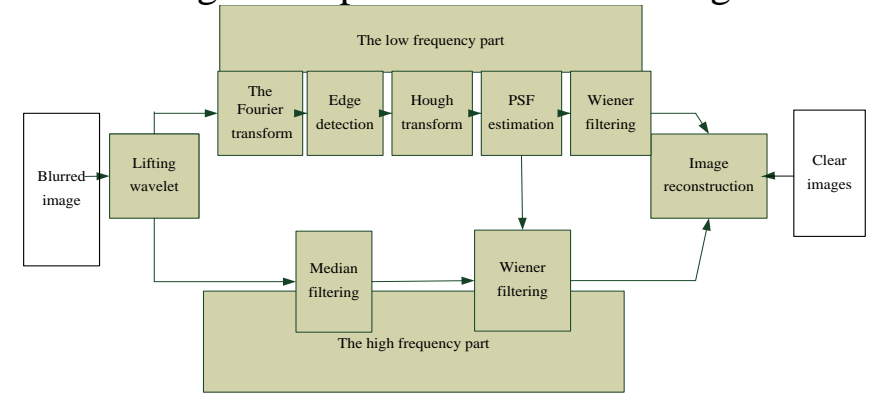

Fig. 1 Terminal guidance phase motion blur image restoration algorithm process 


\section{Frequency domain estimation method of motion blurred image of PSF improvement}

Daubechies wavelet belongs to the biorthogonal wavelet, biorthogonal wavelet with linear phase and is widely used in image processing field, with the improved Daubechies9/7 improve wavelet method, the original single frame motion blurred image are decomposed. Due to the fuzzy information of the image mainly exist in the accounts for most of the energy of the low frequency part of image, so the wavelet transform after ascending the low-frequency part of PSF frequency domain estimation, can avoid the high frequency noise, get more accurate motion blurred image PSF.

Set more than one light spots are $P_{i}(\rho, \theta), i=1,2 \ldots \mathrm{N}$, where $\mathrm{N}$ represents for point number. Take the value of the average of all light spots as threshold segmentation, namely:

$$
T=\frac{\sum_{i=1}^{N} P_{i}(\rho)}{N}
$$

Take threshold segmentation as the separating line, and divide the collection of light spots $P_{i}(\rho, \theta) \quad(\boldsymbol{i}=1,2 \ldots N)$ to up and down collections as $\operatorname{Pup}_{m}(\rho, \theta)$ $(m=1,2 \ldots M, M<N)$ and $P_{d o w n}(\rho, \theta)(\mathrm{n}=1,2 \ldots N-M)$, and then take the values of of each collections as and, the difference of the two parameters are the final stripe spacing $\mathrm{S}$ as follows,

$$
\begin{gathered}
\text { Pupmean }=\frac{\sum_{m=1}^{M} \operatorname{Pup}_{m}(\rho)}{M} \\
\text { Pdownmean }=\frac{\sum_{n=1}^{N-M} \operatorname{Pdown}_{n}(\rho)}{N-M} \\
S \approx \mid \text { Pupmean }- \text { Pdownmean } \mid
\end{gathered}
$$

When the space $\mathrm{S}$ between two straight lines is small, the blurring length is larger, it's easy to have a pathological problem, and the algorithm error exists, will also affect the estimation precision of fuzzy length, therefore, a fixed parameter $\mathrm{R}$ which is greater than zero should be added in the valuation formula of the denominator position. For the $\mathrm{V} \times \mathrm{V}$ size blurred image, the motion blurred image fuzzy valuation formulas for length is finally get as

$$
L \approx \frac{V}{S+R} \approx \frac{V}{\mid \text { Pupmean }- \text { Pdownmean } \mid+R}
$$

Corresponding, the determination of fuzzy angle $\alpha$ is indeed directly choosing the average values of the light spots $P_{i}(\rho, \theta)(i=1,2 \ldots N)$ and do some error correction

$$
\alpha \approx \frac{\sum_{i=1}^{N} P_{i}(\theta)-R}{N}
$$

After many experiments, the correction parameter $\mathrm{R}$ of $\sqrt{2}$ is in the most close to the true value.

\section{The high frequency part of ascension based wavelet median filtering de-noising}

TV guided missile terminal guidance phase of visible light video image would inevitably affected by noise, causing images quality, which must deal with the noise. Images after wavelet decomposition the low frequency part and high frequency part is available, the low frequency part represents the profile of the image, the high frequency part shows the details and mixed with noise, therefore, the noise of image is only done to high frequency coefficients with the quantized treatment .

Considering that the noise is often characterized by isolated pixels in the image gray mutation, high-frequency characteristics and spatial correlation, and the image edge of the high frequency 
information is has certain relevance, here we use the wavelet decomposed high frequency part of ascension median filtering de-noising processing.

After denoising of the high frequency part, the motion blur image restoration can be done according to the low frequency part estimation of PSF. The classic wiener filtering method overcome the ill-posed problem which may occur in the process of recovery, can have very good inhibitory effect on the noise amplification, as much as possible to reduce the noise in the output signal components, and recover useful signal at the same time.

The image of high and low frequency transform will be effective due to the lifting wavelet, the high frequency noise concentrate on the high frequency part of image. The signal noise ratio (SNR) of low frequency part is high, the SNR of high frequency part after median filtering is lower compared with the low frequency part, so the wiener filtering recovery is done for high frequency part with different parameter $r$, it has been verified by many experiments that when the high frequency part $r$ take 0.009 , the recovery effect is the best while the low frequency part $r$ equals 0.001 ,. Finally, the $9 / 7$ lifting wavelet inverse transformation is done for each sub recoveries results, and the target restored images are clear.

\section{The simulation experiment and comparative analysis}

Use the method described in this article with the aid of MATLAB 7.1 software, a frame of video image sequence motion blur image restoration is done to a certain type of television guidance missile terminal guidance phase of visible light, and the simulation results are as follows:

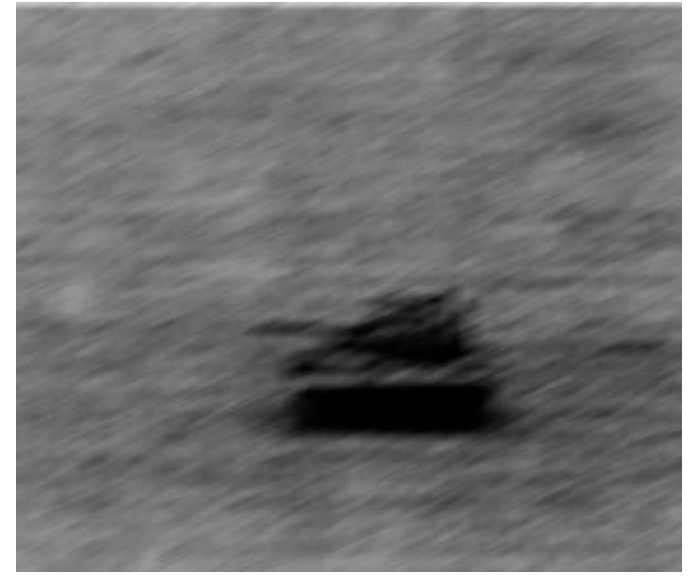

Fig.2 The original motion blurred image

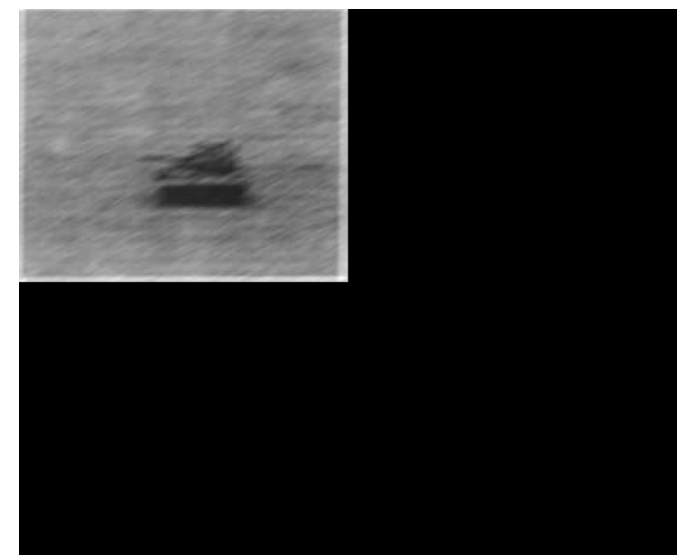

Fig.3 images after lifting wavelet transform

Fig. 2 is the original motion blur the image, Fig. 3 is the image for 9/7 3 lifting wavelet transform images .It shows in Fig. 3 that after lifting wavelet transform, the low frequency part separates the high frequency noise well and save most of the blurred image information. 


\section{Conclusion}

According to the characteristics of dynamic frame motion blurred image of the TV guided missile, the dynamic image frame motion blur recovery method are proposed based on the 9/7 lifting wavelet method. The frequency domain of PSF estimation is done for the low frequency part, and the valuation method was improved effectively, denoising and median filtering in high frequency part and high frequency subband corresponding wiener filtering method is used to recover. The simulation proved that this method can effectively recover the dynamic image of TV guided missile frame fuzzy.

\section{References}

[1] Bingren Zhang, Liming Chen: Mass reduction process of motion blurred image analysis and recovery technology research [J]. Journal of Image and Graphics. Vol.9 (2004) No.7, p.815-819.

[2] Defeng Zhang: MATLAB wavelet analysis [M] (in Chinese). Beijing: China Machine Press. Vol.1 (2009), p.210-219.

[3] Qianrong Chen, Qisheng Lu. Motion blurred image point spread function scale to identify [J]. Computer engineering and application. Vol.23 (2004), p.15-19.

[4] Mingxi Li, Hanping Mao: Based on lifting wavelet transform and median filter in image denoising method research [J]. Journal of laser and infrared. Vol.5 (2007) No.10, p.1109-1111.

[5] Yuanhao Wu, Qian-yang Yu: Based on the Fourier phase difference of the displacement of resisting noise estimation algorithm [J]. Optical precision engineering. Vol.5 (2007) No.7, p. 1138-1142.

[6] Zhengxing Cheng, Shouzhi Yang, Xiaoxia Feng: Theory and algorithms of wavelet analysis, progress and application [M]. Beijing: national defense industry press, Vol.7 (2007), p.38-68.

[7] Zheng Fu, Lixia Xiong, Xiaofeng Zhang: The recovery of digital image processing [J]. Computer and modern. Vol.99 (2003) No.11, p.12-13. 